\title{
MIGRATION OF A CHARGED PARTICLE IN A NON-UNIFORM SINUSOIDAL FIELD: MECHANISMS IN LIQUID AND GASEOUS MEDIA, OPTIMIZATION
}

ALLEN L. SHALOM and FRANCIS LANCELOT

Electrochemistry Dept., Ecole des Mines, 158 Cours Faurie1, 42023 ST-ETIENNE, France and

ISRAEL J. LIN

Mineral Engineering Department

Israel Institute of Technoology, 32000 HAIRA, Israel.

The use of a.c. fields to act upon charged particles is somewhat controversial. Apparently no great effect is expected apart from an oscillation but in fact a migration will occur under certain conditions. When the field is non-uniform and particles are large enough to have inertia, a repulsion force is generated which pushes the particle towards a zone of lower field intensity. Mentions of this possibility can be found in Poh1 (1978) for particles in liquids. For gases it is better known since research has been done on a.c. electrostatic precipitation which presents certain advantages over the classic d.c. type (Erlich and Melcher, 1984). Another group is working on applications that present an interest both in a liquid or gaseous medium. These are the extensive studies of Masuda et al. (1970, 1971, 1974) about the use of various configurations of non-uniform a.c. fields to classify, transport or contain particles. The purpose of this paper is to attempt to understand more thoroughly the mechanism and apply the technique to possible applications in liquid and gaseous media. Thus a simulation will be performed using for simplicity sake the classical case of coaxial electrodes, the calculations are geared to describe trajectories and to predict the optimum frequency for fastest migration.

A general-equation of motion for a particle in a sinusoidal field can be written as follows:

$$
m r^{\prime \prime}+6 \pi \mu a r^{\prime}=q E \cos (\omega t+\phi)+F_{e x}
$$

where $\mathrm{m}=$ particle mass

$a=$ particle radius

$\mu=$ viscosity of medium

$r=$ position vector

$\omega=$ angular frequency

$\mathrm{q}=$ particle charge

$\mathrm{E}=$ peak value of field

$F_{\text {ex }}=$ external force (gravitational, magnetic or drag due to fluid flow).

To simplify the study the external force will be neglected since it adds no interesting effect for the mechanisms investigated. The electric field for the coaxial configuration can be expressed as follows:

$$
E=r \frac{V}{\ln \left(r_{0} / r_{i}\right)}
$$




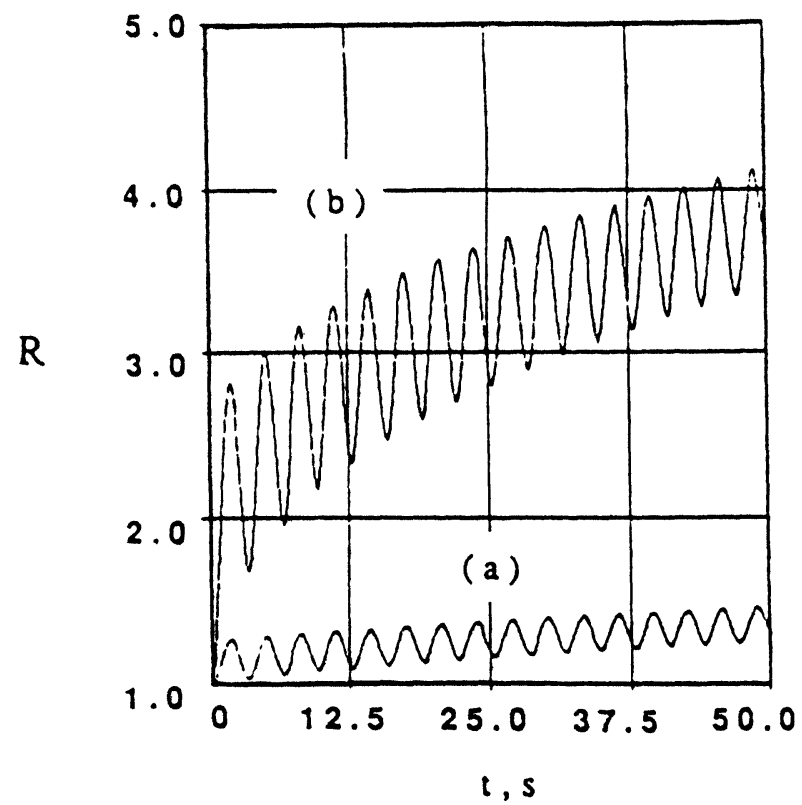

Fig. 1 Migration for two values of $\mathrm{K}$ liq at $\omega=2$ and $\phi=\pi / 2 \quad$ (a) 1.385 (b) 13.85 
where $V$ is the potential difference between the central and external electrodes, $r_{0}$ and $r_{i}$ are their radii respectively. Assuming spherical particles and normalizing $r$ with $r_{i}$ the following more explicite equation is obtained:

$R^{\prime \prime}=\frac{9 \mu}{2 a^{2}\left(\rho_{p}+\rho_{f} / 2\right)} R^{\prime}+\frac{3 q V}{4 \pi r_{i}^{2} a^{3}\left(\rho_{p}+\rho_{f} / 2\right) \ln \left(r_{o} / r_{i}\right)} \frac{\cos (\omega t+\phi)}{R}$

where $R$ is the normalized position vecteur, $\rho_{p}$ and $\rho_{f}$ the particle and fluid densities respectively. To have a clear idea of the meaning of Eq. 3, it can be put in a simpler form:

$$
R^{\prime \prime}=-f_{m} R^{\prime}+K \cos (\omega t+\phi) / R
$$

where $f_{m}$ is a mechanical relaxation frequency-produced by the ratio of drag to inertial forces, $K$ is the ratio of coulombic to inertial forces. Thus the position of the particle can be investigated keeping these two quantities constant and increasing $\tau$, additional variables are $\omega$ and $\phi$. A more useful expression for $K$ is obtained by substituting a proper expression for $q$, depending on the nature of the fluid, i.e., liquid or gas. For liquids, according to the relevant literature:

$$
q=4 \pi \varepsilon_{o} \varepsilon_{f} \zeta a(1+k a)
$$

where $\varepsilon_{0}$ is the vacuum absolute dielectric permittivity, $\varepsilon_{f}$ the relative permittivity of the fluid $\zeta$ the zeta potential and $k^{-1}$ the electrical double layer thickness. In general it can be assumed that $k a<<1$ so after substitution of $q$ in Eq.4, the expression for the force ratio is finally obtained:

$$
\mathrm{K}_{1 i q}=-\frac{3 \varepsilon_{o} \varepsilon_{f} \zeta V}{r_{i}^{2} a^{2}\left(\rho_{p}+\rho_{f} / 2\right) \ln \left(r_{o} / r_{i}\right)}
$$

For a particle in a gas the maximum charge is given as:

$$
\mathrm{q}_{\max }=4 \pi \varepsilon_{0} \varepsilon_{f} \mathrm{p} a^{2} \mathrm{E}_{\mathrm{c}}
$$

where:

$$
p=2 \frac{\varepsilon_{p}-\varepsilon_{f}}{\varepsilon_{p}+2 \varepsilon_{f}}+1
$$

with $1<p<3$ according to the value of $\varepsilon_{p}\left(\varepsilon_{f}\right.$ is practically the same for all gases and close to 1 ). It is of interest to note that Eq. 8 is also valid for conductive particles, for which the permittivity is taken as infinite yielding $p=3 . E_{c}$ is the charging field which cannot be more than the breakdown value for the gas (this value is above all dependent upon the humidity of the gas). For air the highest value is $3.10^{6} \mathrm{~V} / \mathrm{m}$. Thus combining Eq.7,8 


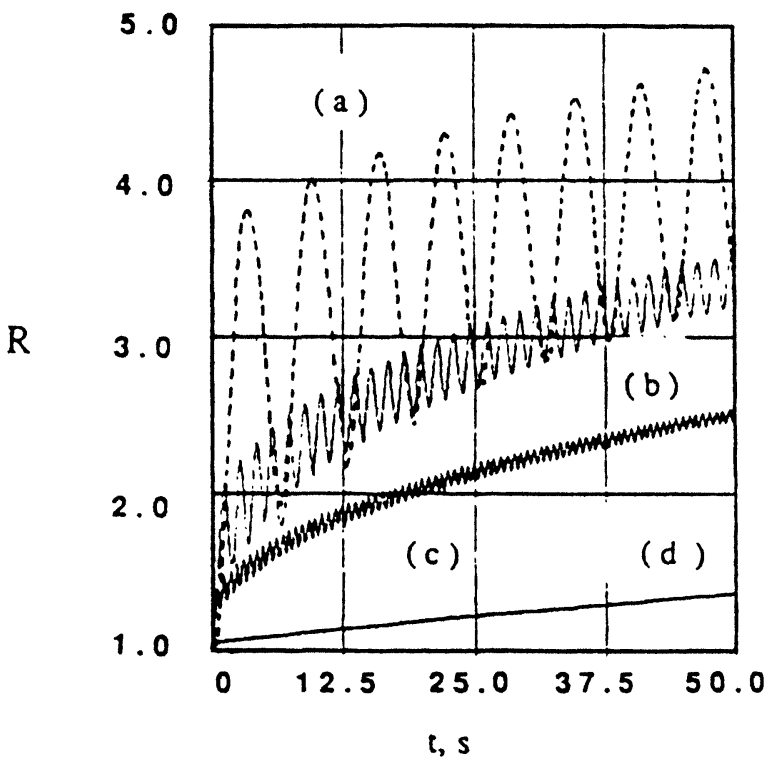

Fig. 2 Effect of frequency ( $\mathrm{rad} / \mathrm{s}$ ) for $\mathrm{K}$ liq $=13.85$

$$
\text { and } \phi=\pi / 2 \quad \text { (a) } 1 \text { (b) } 4 \text { (c) } 10 \quad \text { (d) } 50
$$




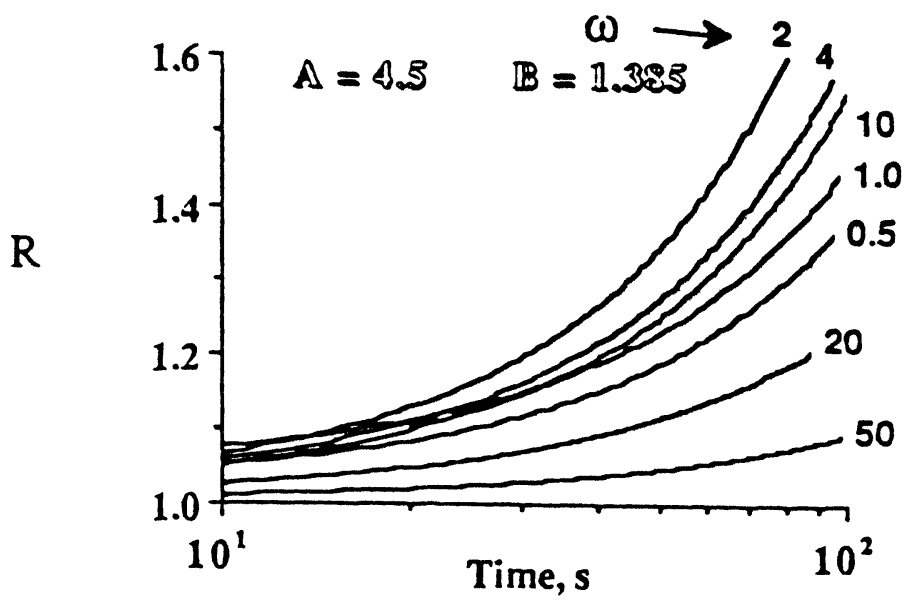

Fig. 3 Migration for $V=100 \mathrm{~V}$

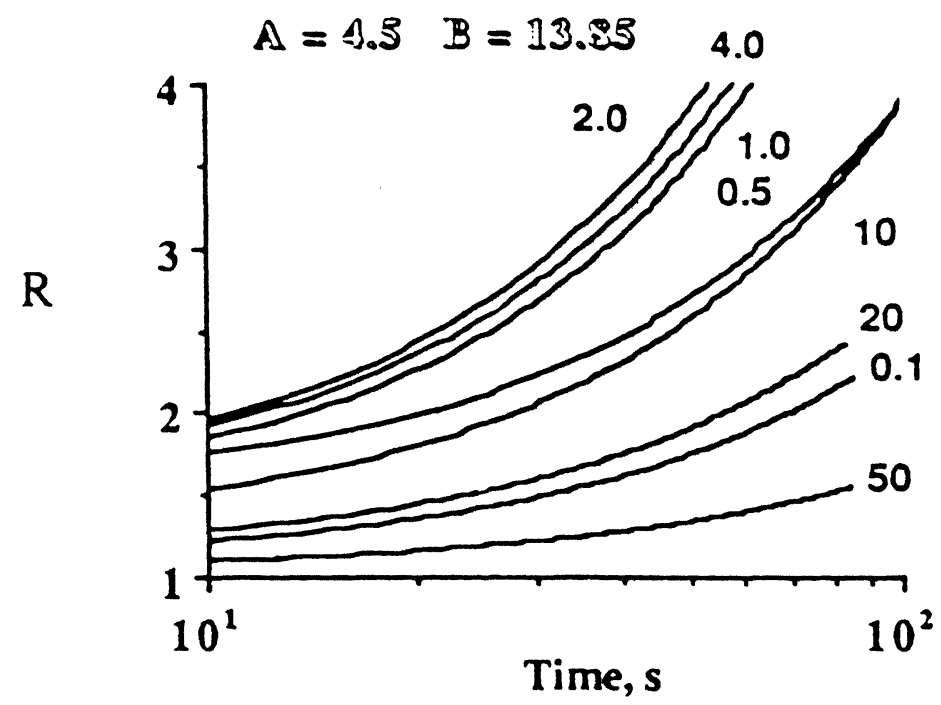

Fig. 4 Same for $V=1000 \mathrm{~V}$ 
and taking in consideration that for gases $\rho_{p}+\rho_{f} / 2 \cong \rho_{p}$, $K$ becomes:

$$
K_{g a s}=\frac{3 \varepsilon_{0} \varepsilon_{f} p E_{c} V}{r_{i}^{2} a \rho_{p} \ln \left(r_{o} / r_{i}\right)}
$$

Calculations have to be done with numerical integration since Eq.4, has no analytical solution. These were performed on an IBM-PC with a software program called ACSL which allows to compute with various methods of integration and gives a graphic output. For this work the Adams-Moulton method with variable step was used because it was found to be the most reliable. The initial conditions were as follows:

$$
t=0 \quad R=1 \quad R=0
$$

Values for the different parameters were chosen to represent a possible separation occurence in deionized water (very low conductivity). The constants are listed below with the resulting value of the parameters in Eq.4:

$$
\begin{array}{lll}
\mu=10^{-3} \mathrm{~Pa} . \mathrm{s} & a=10^{-3} \mathrm{~m} \quad \mathrm{~V}=100 \mathrm{~V} \quad \rho_{\mathrm{p}}+\rho_{\mathrm{f}} / 2=2.10^{3} \mathrm{~kg} \cdot \mathrm{m}^{-3} \\
\varepsilon_{\mathrm{f}}=80 & \zeta=3.10^{-2} \mathrm{~V} \quad \mathrm{r}_{\mathrm{i}}=10^{-3} \mathrm{~m} \quad \mathrm{r}_{\mathrm{o}}=10^{-2} \mathrm{~m}
\end{array}
$$

Thus: $f_{m}=4.5$ and $K_{1 i q}=1.385$

Fig. I shows the effect obtained on the particle for two potential differences $(V=100$ and $1000 \mathrm{~V}$ ). The migration is clearly seen, weakly on the first and strongly on the second, where a non-linear migration velocity is observed. This might be expected since the field is decreasing rapidly away from the central electrode. In Fig. 2 the effect of the frequency is demonstrated keeping everything else constant. There appears to be an optimum frequency for the migration velocity, this frequency is clearly very low.

In Fig. 3 and 4 the plots of the migration velocity against time show a maximum velocity for $\omega=2$. This frequency appears like a resonance frequency of the system and apparently is not much dependent upon the field intensity but more calculations are necessary to know that for sure.

Applications in gases are much easier to realize since the viscosity is about 200 times less than water and $\mathrm{K}_{\text {gas }}$ would be about 1000 times $\mathrm{K}_{1 \text { ig }}$ taking the same data as in the preceding example and a charging field of $10^{6} \mathrm{~V} / \mathrm{m}$. 


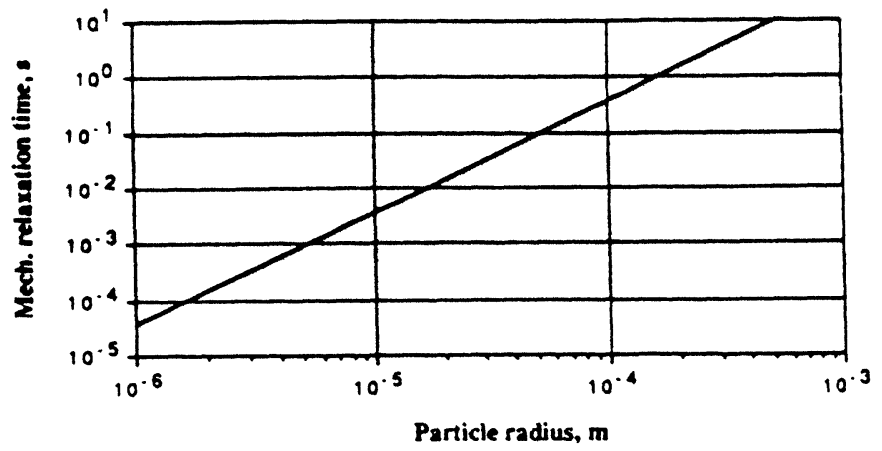

Fig. 5 Plot of mechanical relaxation time vs. particle radius

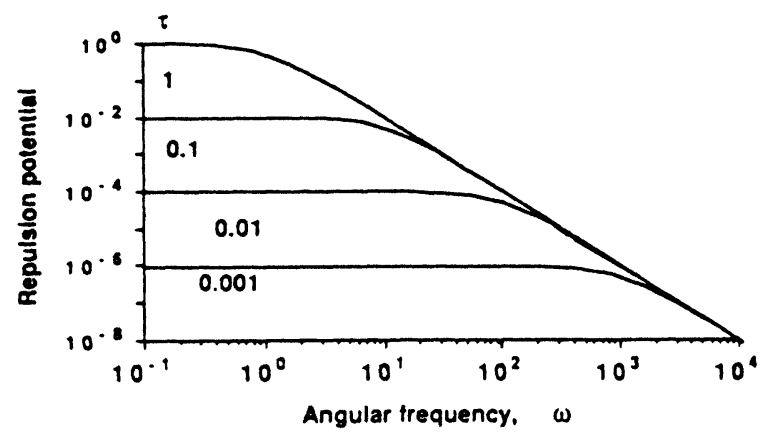

Fig. 6 Repulsion potential dependence upon frequency for various values of particle relaxation time. 


\section{REFERENCES:}

1. M. Ayohama and S. Masuda, Electr. Eng. in Japan, 90(3), 1970-10.

2. R.M. Erlich and J.R. Melcher, Proc.of 1984 IEEE/IAS Ann. Conf., Chicago, 1207.

3. R.M. Erlich and J.R. Melcher, Ibidem, 1211.

4. S. Masuda, "Electric Curtain for Confinement and Transport of Charged Aerosol Particles', Albany Conf.on Electrostatics, June, 1971.

5. S. Masuda and Y. Matsumoto, Electr.Eng. in Japan, 94(6), 1974, 20.

6. H.A. Poh1, "Dielectrophoresis", Cambridge Univ. Press, 1978. 